\title{
Théorie de la régulation et développement durable : essai d'analyse de la diversité nationale en matière d'environnement et de politiques environnementales
}

\author{
Régulation theory and sustainable development: \\ analysis of national diversity of environmental \\ profiles and environmental politics
}

Philippe Lardé et Bertrand Zuindeau*

CLERSÉ UMR 8019

Faculté de sciences économiques et sociales, Université de Lille 1

59655 Villeneuve d'Ascq cedex

\begin{abstract}
Résumé
En dépit de travaux récents cherchant à tester la pertinence de la théorie de la régulation au domaine de l'environnement, il manque de tentatives envisageant une validation empirique de cet apport. L'article souhaite s'inscrire dans cette veine en mettant l'accent sur l'analyse de la diversité des situations et des politiques dans le domaine de l'environnement. Nous recourons à des données relatives aux pays de l'OCDE, traitées par analyse en composantes principales et méthodes de classification. La typologie de pays obtenue est rapprochée d'autres travaux classificatoires plus généraux, notamment Les cinq capitalismes d'Amable (2005). Points communs et différences sont alors passés en revue. L'article s'achève par l'indication de perspectives.
\end{abstract}

() 2010 Lavoisier, Paris. Tous droits réservés.

\footnotetext{
*Adresse email : bertrand.zuindeau@ univ-lille1.fr doi:10.3166/ges.12.261-278 @ 2010 Lavoisier, Paris. Tous droits réservés.
} 


\begin{abstract}
Summary
Despite recent studies seeking to test the relevance of Régulation theory in the environmental field, there are few attempts at making an empirical validation of these contributions. The article would like to do this by emphasising the analysis of the diversity of profiles and policies in the environmental domain. We use data relating to OECD countries, analytically processed into the main components and methods of classification. The typology of the country obtained is compared with other, more general classificatory studies, notably Amable's "five capitalisms" (2005). Points in common and differences are then reviewed. The article concludes with an indication of future prospects.

(C) 2010 Lavoisier, Paris. Tous droits réservés.
\end{abstract}

Mots clés : environnement, politiques environnementales, théorie de la régulation, variété des capitalismes

Keywords: environment, environmental policies, Régulation theory, varieties of capitalisms

\title{
Introduction
}

Depuis plusieurs années, parmi les réponses hétérodoxes aux interrogations théoriques sur l'environnement et le développement durable, la théorie de la régulation a suscité des contributions de plus en plus nombreuses (Gibbs, 1996, 2006 ; Becker, Raza, 2000 ; Gendron, 2001 ; Rousseau, 2002 ; Rousseau, Zuindeau, 2007 ; Zuindeau, 2001, 2007, 2009). En cela, ces contributions répondent à une importante carence de la théorie en question qu'avait soulignée et tenté d'expliquer Alain Lipietz au milieu des années 90 (Lipietz, 1995) : jusqu'alors la théorie de la régulation s'était vraiment peu souciée de la problématique environnementale, question jugée très périphérique au regard du référentiel fordiste. Ces apports régulationnistes plus ou moins récents sur l'environnement et le développement durable, et leur intérêt incontestable, n'empêchent pas une double insuffisance.

D'une part, les travaux sont relativement déconnectés les uns des autres (Zuindeau, 2007). Pour le moins, ils ne forment pas une théorie régulationniste de l'environnement qui aurait sa cohérence, couvrirait largement le domaine concerné et pourrait d'ores et déjà prétendre à être stabilisée. Un tel travail d'harmonisation, d'extension et de stabilisation constitue un important projet heuristique à réaliser dans les années qui viennent.

D'autre part, les applications empiriques des constructions théoriques envisagées sont très réduites. Si l'on excepte quelques contributions isolées relatives à la biodiversité (Görg, Brand, 2000), l'exploitation forestière (McManus, 2002) ou le rapport des entreprises à l'environnement (Gendron, 2001), prenant plutôt la forme de monographies qualitatives, on peut dire que le travail de validation empirique reste, dans une large mesure, à réaliser. L'aile environnementale du corpus régulationniste achoppe ainsi sur une limite qui est parfois énoncée à l'encontre de cette même théorie dans son ensemble : comment fonder empiriquement ce qui, à défaut, demeure à l'état d'hypothèses relatives aux caractéristiques et à l'évolution des régimes d'accumulation et des modes de régulation?

C'est plus particulièrement à ce manque empirique que le présent article cherche à apporter réponse, réponse très partielle, cependant, et appelée à être confortée.

Comment avancer en la matière ? Si a priori, comme pour tout travail empirique, les procédures de validation sont susceptibles de prendre des formes multiples (analyse 
qualitative, approches statistiques et économétriques, etc.), il nous semble que la théorie de la régulation suscite plus particulièrement deux voies opportunes (Zuindeau, 2007) :

- l'approche historique, d'une part. Le corpus régulationniste est familier des analyses de temps long, portant sur un territoire particulier (pays ou territoire infranational). Les exemples de monographies sont multiples, montrant comment une économie a évolué dans le temps, passant d'un régime d'accumulation à un autre, modifiant plus ou moins sensiblement ses formes institutionnelles et son mode de régulation. Appliqué à la problématique environnementale, ce type de traitement consisterait à voir comment le « rapport économique à l'environnement $»^{1}$ (Rousseau, 2002 ; Zuindeau, 2007, 2009) évolue lui-même dans le temps long en lien avec des caractéristiques socioéconomiques générales ;

- l'approche comparative, d'autre part. La théorie de la régulation attache aussi un intérêt particulier aux comparaisons, notamment entre économies nationales (Théret, 1997 ; Boyer, 2002 ; Amable, 2005). La dimension comparative est même au cœur du programme de recherche de cette théorie. En reconnaissant une variété de formes au capitalisme et en offrant une analyse de cette variété, les auteurs régulationnistes sont ainsi plus à même de contester l'idée d'une universalité des « bonnes politiques ». Au contraire, comme les formes institutionnelles et leur mode d'agencement sont différents d'une économie à l'autre, il est alors possible de justifier que les politiques économiques pertinentes sont elles-mêmes variables selon cette variété de formes.

Sans bien sûr dénier l'intérêt de la première voie, notre contribution souhaiterait permettre une avancée selon la seconde : l'approche comparative entre économies nationales, des situations et politiques environnementales. La notion d'environnement est ici entendue dans l'acception relativement limitée d' " environnement naturel » et correspond à des éléments de nature - tels que l'air, l'eau, l'énergie, les aménités du paysage, certaines fonctions écosystémiques de la nature -, servant de ressources économiques et de biens de consommation, mais a à voir aussi avec les questions de déchets et de pollution, ainsi qu'avec l'espace de localisation des individus et des activités. Les « politiques environnementales » désignent, quant à elles, les formes d'action, principalement mais pas exclusivement publiques, visant à prévenir, réduire et traiter les pollutions, à économiser les ressources naturelles, et plus largement, à préserver la qualité de l'environnement. Du point de vue de son contenu empirique, l'article a pour objet :

de traiter par méthodes d'analyses de données (analyse en composantes principales et méthode des nuées dynamiques) les variables environnementales et de politiques environnementales des pays de l'OCDE ;

d'établir une (ou des) classifications des pays concernés sous l'angle environnemental ; de rapprocher ces classifications de typologies plus générales, établies dans le cadre d'analyses d'inspiration régulationniste (Amable, 2005);

d'esquisser des premiers éléments d'explication des positionnements obtenus.

Ces divers aspects seront présentés dans la principale section du texte, la section 2 cen-

\footnotetext{
${ }^{1}$ Autrement dit, l'ensemble des relations économiques liant les caractéristiques du régime d'accumulation et du mode de régulation, d'un côté, et l'environnement naturel, de l'autre. Précédemment (Zuindeau, 2007, 2009), nous avons tenté d'explorer l'hypothèse que, dans un contexte capitaliste donné, le rapport économique effectif que l'homme entretient avec son environnement naturel est la résultante de trois formes fondamentales : une forme élémentaire (ou «transhistorique » au sens de Ted Benton, 1989), une forme capitaliste générale et une forme capitaliste particulière liée au régime d'accumulation/mode de régulation particulier.
} 
trée sur le travail empirique (méthodes mobilisées, résultats, éléments de comparaison). Au préalable, la section 1 a pour objet la reprise de la question comparative vue à la lumière de la théorie de la régulation et d'envisager une possible extension à la problématique environnementale.

\section{Diversité géographique des formes du capitalisme sous l'éclairage de la théorie de la régulation : vers une prise en compte de l'environnement}

Institutionnalisme historique (Théret, 2000), la théorie de la régulation est, par là même, critique de toute construction analytique qui prétendrait rendre compte de la réalité économique à l'aide de lois de portée universelle, sans référence particulière à la diversité géographique et à l'histoire des contextes socioéconomiques. On comprend dès lors que la théorie néoclassique est tout spécialement visée par le feu d'une telle critique. Au contraire, chez les régulationnistes, la variabilité est une question importante. La variabilité ne concerne pas ou pas seulement les inévitables variations quantitatives des justement appelées variables économiques - le reconnaître relèverait du truisme -, mais s'applique surtout aux régularités entre ces variables ainsi qu'à leurs modes particuliers d'articulation.

Dans cet esprit, deux démarches peuvent s'envisager. La première est inductive. Elle procède de la diversité des configurations socioéconomiques observables d'un territoire à l'autre - notamment des espaces nationaux - et cherche à voir ce qui rapproche ou distingue les territoires en question. À la limite, on aboutit à une ou des typologies de territoires. L'autre démarche consiste à partir d'un modèle théorique - par exemple une certaine configuration socioéconomique qu' on appellera le fordisme - pour juger de l'importance de sa diffusion en le rapprochant de la réalité des situations selon les territoires. Ce faisant, un tel rapprochement entre un ensemble de relations, posé à titre d'hypothèses, et la réalité de certains modes d'agencement prend la forme d'une validation empirique.

Au total, dans un cas comme dans l'autre, il s'agit de comparer. Et, une fois encore, la nécessité de cette épreuve comparative est d'autant plus grande que l'on admet la prémisse d'une diversité fondamentale des formes économiques et non l'existence d'anomalies plus ou moins gênantes par rapport à un schéma universel.

Sur la base de cette orientation méthodologique, en particulier dans l'optique de la première voie décrite, la théorie de la régulation est à l'origine d'un certain nombre de travaux de comparaisons internationales, lesquels aboutissent à des typologies du capitalisme (pour une synthèse, voir Boyer, 2002, notamment le tableau synoptique des pages 134-135). Une des tentatives les plus récentes et les plus ambitieuses en la matière est celle de Bruno Amable, publiée dans son ouvrage Les cinq capitalismes (2005)².

\footnotetext{
${ }^{2}$ À dire vrai, le rattachement de ce travail d'Amable à la théorie de la régulation est contestable et pourrait surtout être contesté par l'auteur lui-même ! - car s'il est fait référence, dans l'ouvrage, à des contributions antérieures explicitement régulationnistes, notamment avec Amable comme co-auteur (Amable et al., 1997), l'auteur semble pourtant se démarquer de ce courant par une construction plus personnelle. En particulier, il ne se laisse pas contraindre par les « formes institutionnelles » traditionnellement retenues par les régulationnistes et privilégie d'autres « domaines institutionnels » (p. 124), la protection sociale et le secteur éducatif. Cependant, outre que ces autres domaines ont été également investis par des auteurs régulationnistes (Bruno Thérêt, Ève Caroli, Thomas Lamarche...), les présupposés épistémologiques d'Amable (dimension historique, importance des institutions, etc.) laissent penser que la communauté de réflexion avec l'École de la Régulation est plus qu'admissible.
} 
Cet auteur cherche à fonder l'hypothèse d'une partition des pays capitalistes au regard de plusieurs domaines : marché de produits, marchés du travail, finance, protection sociale, éducation, spécialisations scientifiques et techniques. Recourant à des analyses factorielles et classificatoires, il parvient à corroborer sa thèse de cinq grandes formes géographiques de capitalisme (p. 223) : capitalisme libéral de marché (Australie, Canada, Royaume-Uni, USA), capitalisme asiatique (Japon, Corée), capitalisme européen continental (Suisse, Pays-Bas, Irlande, Belgique, Norvège, Allemagne, France, Autriche), capitalisme social-démocrate (Danemark, Finlande, Suède), capitalisme méditerranéen (Grèce, Italie, Portugal, Espagne), même si, comme on pouvait le présumer, on n'obtient pas exactement une telle typologie sur chacun des grands domaines étudiés.

Mais une telle façon de faire ne serait que descriptive si elle ne s'accompagnait d'un registre explicatif, notamment pour comprendre pourquoi les contenus des différentes formes structurelles se correspondent et ne sont pas distribués de manière aléatoire. Pour ce faire, Amable mobilise le concept de « complémentarité institutionnelle », emprunté à Aoki (1994; 2001), qui traduit l'existence d'influences réciproques entre les différentes formes (ou domaines) institutionnels. Plus précisément, « [1]a complémentarité institutionnelle est présente quand l'existence ou la forme particulière prise par une institution dans un domaine renforce la présence, le fonctionnement ou l'efficacité d'une autre institution dans un autre domaine » (Amable, 2005, p. 83). Ainsi, si l'on reprend deux formes institutionnelles retenues par le théorie de la régulation - le rapport salarial et la forme de l'État -, on peut dire, par exemple, que dans le cas du fordisme, la norme salariale a pour corollaire un rôle significatif de l'État dans la définition et la diffusion des revenus indirects. Ou alors - deuxième exemple - dans un système économique de type libéral, la concurrence par les prix sera forte, l'État sera économiquement plus neutre, et s'agissant du régime salarial, la protection des travailleurs sera relativement faible. Il n'y a, certes pas, de déterminations absolues en la matière et la pluralité des configurations reste possible, mais pour autant, cette pluralité n'est pas infinie et le nombre de figures observables demeure limité : d'où les familles d'économies obtenues au travers de typologies.

Le présent travail empirique consiste à appliquer, au domaine de l'environnement, à la fois la réalisation de telles typologies et l'hypothèse de complémentarité institutionnelle. Le premier aspect, quoique rarement traité, comporte quand même des précédents. Ainsi, reprenant un travail de Benhaim et al. (1991), Alain Lipietz, dans un article de 1997, montre comment se positionnent les différents pays (au début des années 90) dans la politique relative au changement climatique. Sur la base de plusieurs critères concernant le niveau de développement atteint, les conditions de production et la consommation énergétique, il apparaît que les différents pays considérés (une centaine) peuvent être classés suivant deux axes principaux : le niveau de développement économique et la contribution, par unité produite, à l'effet de serre. Or, les pays les plus actifs dans la politique de lutte contre l'effet de serre sont ceux qui sont aussi à la fois les plus développés et les plus propres (Scandinavie, Allemagne, Japon), tandis que les États-Unis, par exemple, sont plutôt dans une attitude d'opposition, du fait d'une contribution importante à l'effet de serre et en dépit d'un niveau de développement élevé.

Or, Lipietz retrouve, dans la typologie obtenue, la classification qu'il a par ailleurs établie et théorisée des différentes économies nationales, selon leur démarche de dépassement du fordisme : rigidité ou flexibilité, d'une part, et type d'implication négociée, d'autre part. Loin d'être aléatoire, ou simplement issu de facteurs contingents, le positionnement des 
pays vis-à-vis d'une politique telle que la lutte contre le changement climatique peut être éclairé par des caractéristiques de nature institutionnelle, exerçant un rôle plus général sur la dynamique d'activités et sa régulation. On retrouve là l'hypothèse de complémentarité institutionnelle décrite précédemment et qu'on propose d'appliquer aussi au domaine de l'environnement et des politiques environnementales. Plus précisément, l'idée suggérée ici est que les caractéristiques du rapport économique à l'environnement sont influencées par le contenu et l'évolution des formes institutionnelles, dans une économie donnée.

Ainsi,la forme de l'État, l'une des cinq formes institutionnelles ${ }^{3}$ selon les régulationnistes, influence incontestablement la manière dont se constitue le rapport économique à l'environnement. Par exemple, durant la période fordiste d'après-guerre, le renforcement de l'État-Providence et son rôle général sur l'économie (contribution à la formation et à la stabilisation d'une norme de consommation, via l'extension des formes de salaires indirects, pilotage macroéconomique d'ensemble, etc.) vont trouver aussi à s'appliquer au domaine de l'environnement. D'une part, l'État va agir de manière significative sur l'organisation de l'approvisionnement énergétique. En France, la mise en œuvre de la politique électronucléaire est, de ce point de vue, à mentionner tout particulièrement. D'autre part, l'État ainsi que les collectivités locales vont s'employer à gérer les problèmes d'externalités touchant aux domaines de l'eau et des déchets. La mise en place des réseaux d'adduction d'eau et de collecte des eaux usées, de même que la collecte et le traitement des déchets, vont fortement progresser après la seconde guerre mondiale, cherchant à offrir aux hommes et aux activités un contexte environnemental plus satisfaisant ${ }^{4}$.

Les caractéristiques de la concurrence, autre forme institutionnelle, fournissent une deuxième illustration de complémentarité associant l'environnement. Quand la concurrence s'exerce essentiellement via les coûts, les freins à l'internalisation des externalités environnementales sont nombreux et les stratégies volontaristes des entreprises en faveur de l'environnement demeurent très limitées. A contrario, quand les formes de la concurrence s'orientent vers des composantes « hors-coûts » (qualité, réactivité, flexibilité, etc.), les politiques environnementales sont mieux acceptées par les entreprises et des formes d'auto-contrôle (reporting environnemental, management environnemental, RSE, etc.) se développent.

Dernier exemple, ayant trait aux modes d'insertion à l'économie internationale : une économie qui s'ouvre progressivement sur l'économie mondiale aura pour souci, ou de limiter la sévérité de ses normes nationales pour éviter une érosion de sa compétitivité, ou de rechercher une coopération avec d'autres pays pour aboutir à des normes partagées sur le plan international. Pour illustrer le premier terme de l'alternative, il est possible de faire référence aux atermoiements et même reculs dans le domaine de la lutte contre le changement climatique, laquelle, à tort ou à raison, est suspectée de porter préjudice à la compétitivité des entreprises 5 .

\footnotetext{
${ }^{3}$ Rappelons-les, en nous contentant d'en mentionner les intitulés : les formes de la contrainte monétaire, les configurations du rapport salarial, les formes de la concurrence, les modalités d'adhésion ai régime international, les formes de l'État (pour une présentation générale, nous renvoyons à l'ouvrage central de la théorie de la régulation : Théorie de la régulation - L'état des savoirs - Boyer, Saillard, 1995).

${ }^{4}$ L'une et l'autre caractéristiques confèrent une certaine spécificité à la France. Dans d'autres pays, la faiblesse du poids voire l'absence complète du nucléaire ont été des facteurs facilitant l'émergence d'une autre configuration énergétique (par exemple les énergies renouvelables au Danemark). Par ailleurs, dans certains pays, les politiques environnementales sont beaucoup plus décentralisées qu'en France : Allemagne, pays scandinaves.

${ }^{5}$ Dernier exemple en date, bien sûr : le renvoi sine die de la mise en œuvre de la « contribution carbone » en France, au motif de l'absence d'instruments équivalents dans les autres grands pays partenaires.
} 
a contrario, pour rendre compte de la diffusion internationale de normes environnementales, on citera l'exemple des référentiels de certification de type ISO14000 dont la progression, au niveau mondial, est assez spectaculaire. Outre que de telles normes traduisent bien les incursions de la dimension « hors-coûts » dans les registres de la compétitivité, leur portée, d'emblée mondiale, favorise indéniablement les échanges de biens entre pays.

Tant s'en faut, le présent article ne permettra pas de réaliser une analyse systématique des relations entre les formes institutionnelles traditionnelles de la théorie de la régulation et les caractéristiques de l'environnement et des politiques environnementales, et ce, selon les divers pays considérés. Un tel travail requerrait un programme de recherche à part entière. L'on se contentera, en sus de l'exercice d'établissement de familles de pays au regard de l'environnement, d'apporter quelques commentaires, encore relativement généraux et provisoires, aidant à éclairer les positionnements obtenus.

\section{Diversité des situations et des politiques environnementales}

Ainsi qu'annoncé dans l'introduction, la présente investigation tente d'appréhender la diversité nationale des situations dans le domaine de l'environnement et des politiques environnementales. Il faut comprendre la notion d'environnement dans une acception relativement limitée d'environnement naturel. Ni ce que l'on appelle habituellement le cadre de vie ni le contexte général dans lequel s'insèrent les activités économiques ne sont ici considérées. Comme on va le voir avec le paragraphe suivant, les situations environnementales sont cernées au travers d'indicateurs clés de pollution et d'utilisation de ressources, tandis que la caractérisation des politiques environnementales passe par l'examen de variables de taxe environnementale et de mode de gestion des déchets. En l'occurrence, comme on le verra, le rattachement à l'une ou l'autre des deux catégories (situations environnementales ou politiques environnementales) ne va pas toujours de soi.

\subsection{Données et éléments de méthode}

Les données sont toutes issues du COMPENDIUM sur l'environnement de l'OCDE (OCDE, 2007). Ont été considérées les variables suivantes : émissions de gaz carbonique par habitant [CO2], émissions d'oxydes de soufre par habitant [oxydes de soufre], émissions d'oxydes d'azote par habitant [oxydes d'azote], émissions de monoxyde de carbone par habitant [CO], utilisation des ressources en eau douce par habitant [eau], déchets municipaux par habitant [déchets municipaux], déchets mis en décharge par habitant [mise en décharge], recyclage des déchets par habitant [recyclage], déchets nucléaires rapportés à l'approvisionnement total en énergie primaire [déchets nucléaires], taxes relatives à l'environnement par rapport au PIB [taxe 1], taxes relatives à l'environnement par rapport à l'ensemble des recettes fiscales [taxe 2]. Le tableau A en annexe précise les valeurs collectées ${ }^{6}$. On remarque ainsi que la base de données comporte des variables de pression sur l'environnement ou d'utilisation de ressources naturelles, ainsi que des variables reflétant les politiques environnementales. En l'occurrence, la répartition des

\footnotetext{
${ }^{6}$ Selon les variables et selon les pays, les dates de valeurs changent : de fin des années 90 à 2005. Comme notre étude s'intéresse aux aspects de structure et non d'évolution, cette variabilité n'est pas vraiment gênante.
} 
variables entre ces trois rubriques n'est pas aussi évidente qu'elle pourrait paraître. Les variables de taxes sont, certes, des variables de politiques environnementales, mais la valeur considérée n'est pas indépendante des volumes de pollutions supportés. De même, les trois variables de déchets que sont « mise en décharge », « recyclage » et « déchets nucléaires » manifestent bien une pression plus ou moins forte sur l'environnement (avec même une corrélation négative s'agissant du recyclage), mais révèlent aussi un type de politiques en cours (politiques énergétiques s'agissant des « déchets nucléaires ») 7 .

Pour comparer les pays, deux techniques d'analyse statistique ont été mobilisées : l'analyse en composantes principales (ACP) et la classification en nuées dynamiques (CND).

L'ACP mettra en évidence les combinaisons de variables - les « composantes principales »-qui différencient le mieux les pays comparés.

La CND regroupera les pays en classes homogènes selon l'impact environnemental et la politique environnementale.

Pour permettre la comparaison avec la typologie d'Amable, l'analyse a porté sur un sous-ensemble de 21 pays de l'OCDE (voir la liste dans le tableau A en annexe). Une extension à l'ensemble des pays de l'OCDE peut évidemment être envisagée.

La suite du texte expose les principaux résultats obtenus, en présentant d'abord ceux relatifs à l'ACP puis ceux issus de la CND.

\subsection{Résultats de l'analyse en composantes principales}

Le tableau 1 résume les résultats de l'ACP (page 269).

Le graphique 1 (page 269) résume les corrélations entre les onze variables et leurs corrélations avec les deux premières composantes ${ }^{8}$. Le graphique 2 (page 270) représente le positionnement des 21 pays sur le plan formé par les deux premières dimensions (premier plan factoriel). Le graphique 3 (page 271) vient nuancer les proximités entre les points-pays du graphique 2 en tenant compte de la troisième composante.

L'interprétation des résultats porte sur les trois premières composantes qui restituent $71,7 \%$ de la variance. La réduction factorielle dans ces trois dimensions est satisfaisante ${ }^{9}$.

La première composante, très prédominante $(43,5 \%$ de la variance expliquée), oppose nettement les émissions de polluants de l'air, l'utilisation des ressources en eau douce, les déchets mis en décharge, aux taxes liées à l'environnement. En d'autres termes, de façon très simplifiée, les pays où l'impact environnemental est le plus fort n'ont pas développé de mesures incitant à sa réduction, en tout cas d'instruments fiscaux ${ }^{10}$.

\footnotetext{
${ }^{7}$ Par ailleurs, trois variables illustratives (ne jouant pas sur la formation des axes) ont été adjointes à l'analyse : densité de population, PIB par habitant, part de l'emploi agricole.

${ }^{8}$ Cf. matrice des composantes en annexe (tableau B)

${ }^{9} \mathrm{La}$ qualité de la réduction factorielle dans les trois premières dimensions est par ordre décroissant : $\mathrm{CO}$ $(0,842)$, recyclage $(0,832), \mathrm{CO}_{2}(0,791)$, déchets nucléaires $(0,784)$, oxydes d'azote $(0,783)$, oxydes de soufre $(0,773)$, taxe $2(0,713)$, taxe $1(0,686)$, eau $(0,609)$, déchets municipaux $(0,599)$, mise en décharge $(0,483)$.

${ }^{10}$ En effet, la base de données rend mal compte de l'arsenal réglementaire dont disposent les États. Notamment, en matière d'incitations financières, les USA recourent aussi aux permis à polluer pour réduire les émissions de dioxyde de soufre et d'oxydes nitreux (Acid Rain Program).
} 
Tableau 1 : Résumé des résultats de l'ACP

\begin{tabular}{|c|c|}
\hline \multicolumn{2}{|c|}{ Caractéristiques des composantes } \\
\hline $\begin{array}{c}\text { Composantes dont la valeur propre est }>1 \\
\text { et } \% \text { de variance expliquée }\end{array}$ & $\begin{array}{l}\text { - Composante } 1: 43,5 \% \\
\text { - Composante } 2: 15,8 \% \\
\text { - Composante } 3: 12,4 \% \\
\text { - Composante } 4: 10,3 \%\end{array}$ \\
\hline $\begin{array}{l}\text { Coordonnées sur la composante } \mathbf{1} \\
\text { (ou coefficients de corrélation avec la } \\
\text { composante } 1 \text { ) }\end{array}$ & $\begin{array}{c}\text { Monoxyde de carbone }(0,883) \\
\text { Oxydes d'azote }(0,876) \\
\text { Oxydes de soufre }(0,856) \\
\mathrm{CO}_{2}(0,795) \\
\text { Eau douce }(0,725) \\
\text { Mise en décharge }(0,515) \\
\text { Taxes/recettes fisc. }(-0,716) \\
\text { Taxes / PIB }(-0,730)\end{array}$ \\
\hline $\begin{array}{l}\text { Coordonnées sur la composante } 2 \\
\text { (ou coefficients de corrélation avec la } \\
\text { composante } 2 \text { ) }\end{array}$ & $\begin{array}{l}\text { Recyclage des déchets }(0,748) \\
\text { Déchets municipaux }(0,746) \\
\text { Déchets nucléaires }(-0,492)\end{array}$ \\
\hline $\begin{array}{c}\text { Coordonnées sur la composante } 3 \\
\text { (ou coefficients de corrélation avec la } \\
\text { composante } 3 \text { ) }\end{array}$ & Déchets nucléaires $(0,736)$ \\
\hline
\end{tabular}

Graphique 1: La projection des onze variables sur le premier plan factoriel

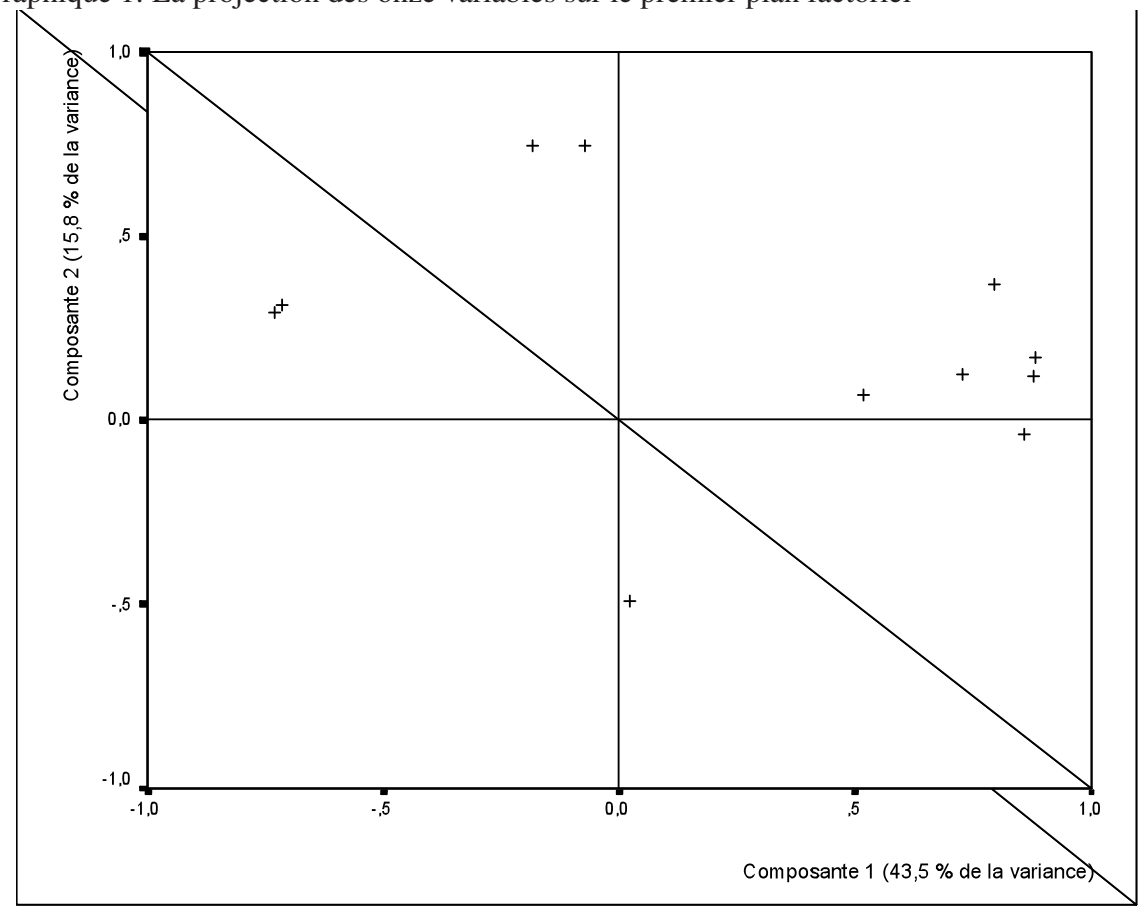


Graphique 2 : La projection des 21 pays sur le premier plan factoriel

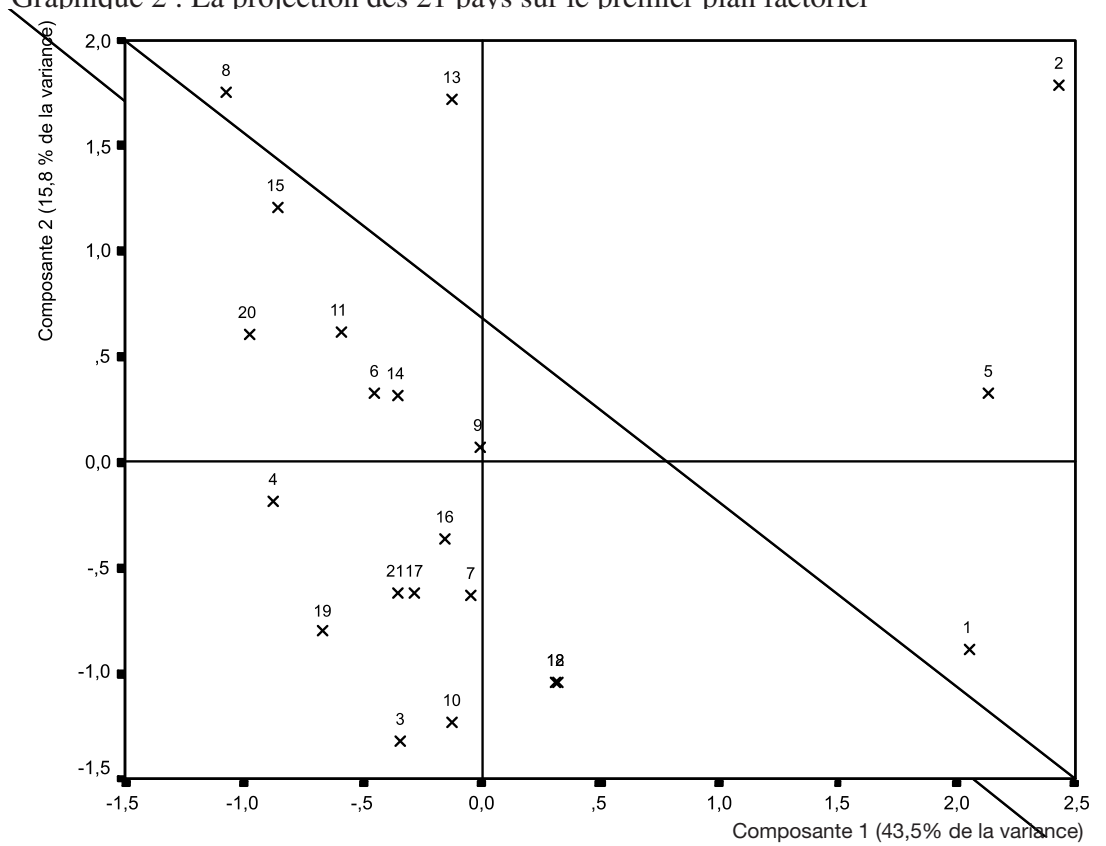

Code des pays :

(1) Canada, (2) USA, (3) Japon, (4) Corée, (5) Australie, (6) Autriche, (7) Belgique, (8) Danemark, (9) Finlande, (10) France, (11) Allemagne, (12) Grèce, (13) Irlande, (14) Italie, (15) Pays-Bas, (16) Norvège, (17) Portugal, (18) Espagne, (19) Suède, (20) Suisse, (21) Royaume-Uni.

Remarque : La Grèce et l'Espagne sont confondues sur le graphique 2.

Graphique 3 : La projection des 21 pays sur le plan formé par les composantes 1 et 3

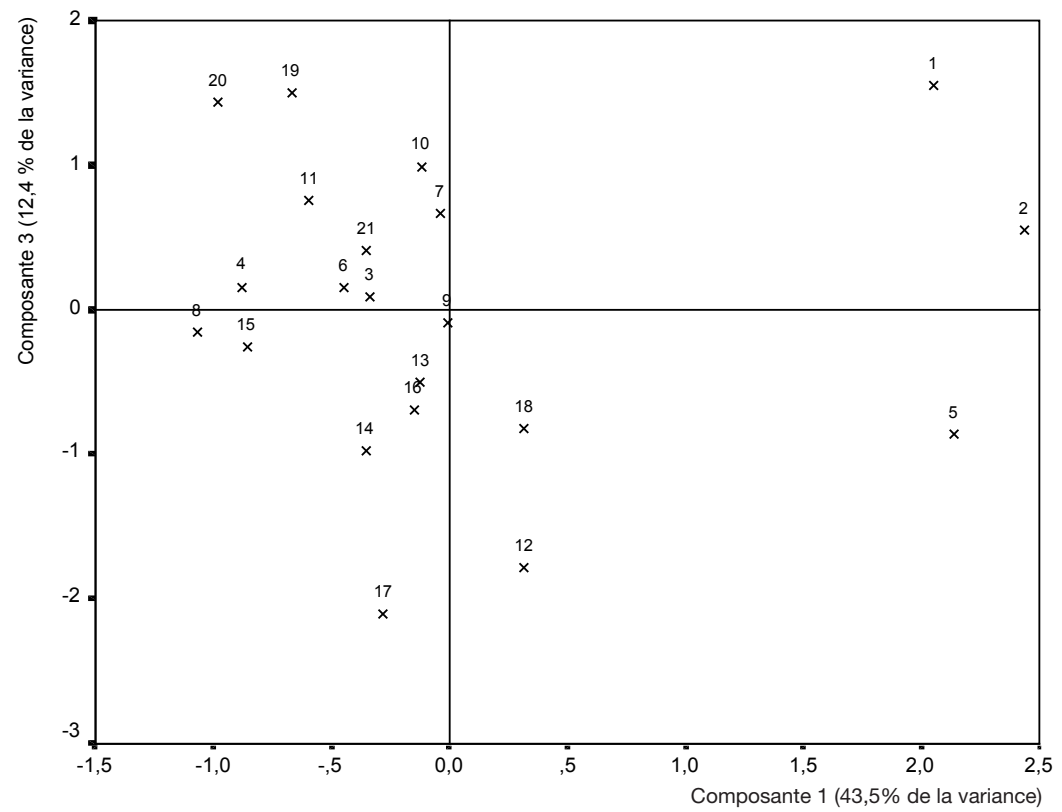


Le positionnement des USA, du Canada, de l'Australie sur la première composante est significatif de niveaux d'émission de polluants de l'air, de consommation d'eau, de mise en décharge, qui sont de très loin les plus élevés et de taxes liées à l'environnement rapportées au PIB (taxe 1) et aux recettes fiscales (taxe 2) les plus faibles. Les données brutes présentées en annexe dans le tableau A expriment, d'ailleurs, directement ce faible poids de la fiscalité environnementale des trois pays susnommés : par exemple, en proportion du PIB, la fiscalité écologique est limitée à $0,9 \%$ aux USA, 1,2 \% au Canada et $1,7 \%$ en Australie, quand les pourcentages atteignent 3,3\% en Finlande, 3,6 \% aux Pays-Bas et jusqu'à 4,8\% au Danemark.

La deuxième composante se construit surtout autour des déchets municipaux et de leur recyclage comme l'indiquent leurs forts coefficients de corrélation avec cette composante ${ }^{11}$. Cette configuration pourrait révéler une opposition entre économies plutôt « riches » (USA, Danemark, Suisse...) et « moins riches » (Grèce, Espagne, Portugal) (voir le graphique 2), s'il n'y avait l'effet un peu perturbateur de la variable « déchets nucléaires » tirant vers le bas des pays tels que le Japon, la France et le Canada (voir tableau A en annexe s'agissant du poids du nucléaire).

Cela étant, l'étirement des axes du graphique 2 illustre la grande hétérogénéité des situations et des politiques environnementales nationales. Trois pays sont très atypiques. Tout d'abord les USA, dont la position est la plus excentrée sur les deux composantes, ont l'impact environnemental de très loin le plus important et le niveau relatif de taxation le plus bas. Puis l'Australie et le Canada qui, à un degré moindre, présentent une configuration analogue, peu vertueuse à l'égard de l'environnement. Le poids de l'énergie nucléaire qui vient d'être évoqué contribue surtout à la constitution de la troisième composante ${ }^{12}$. L'ACP montre que pour ce choix énergétique, il existe un net clivage entre les pays de l'OCDE avec, en haut de l'axe vertical du graphique 3, les pays qui développent une filière nucléaire tels la France, le Canada, la Suède et, en bas de l'axe, ceux qui n'ont pas choisi ce mode d'énergie comme le Portugal, l'Australie, la Grèce (cf. tableau A des données en annexe).

Les proximités (ou ressemblances) des pays dans les deux premières dimensions (graphique 2) sont donc à nuancer par leur éloignement sur le troisième axe (graphique 3). L'Australie et le Canada en sont le meilleur exemple ${ }^{13}$.

\footnotetext{
${ }^{11} \mathrm{Cf}$. tableau 1 . Le deuxième axe factoriel explique $15,8 \%$ de la variance, soit une diminution considérable au regard de la première composante $(43,5 \%)$. Cela peut s'expliquer par le fait que la caractérisation des situations et politiques environnementales est, pour l'essentiel, redevable d'un effet de volume, opposant les impacts polluants (exprimés par habitant) forts et les impacts faibles.

${ }^{12}$ Cette composante représente $12,4 \%$ de la variance.

${ }^{13}$ Notre analyse de données a été complétée par des tests de sensibilité, cherchant à voir si les principaux résultats se trouvaient altérés lorsque telle variable ou tel pays, quelque peu atypiques, étaient omises. Ont, par exemple, été traitées de la sorte la variable « déchets nucléaires » et « l'Australie ». Même si la suppression de ces variables n'est pas sans incidence (par exemple, et à l'évidence, l'omission de « déchets nucléaires » transforme complètement la composante 3), pour autant, les résultats principaux et les enseignements susceptibles d'en être tirés demeurent les mêmes. Par ailleurs, l'introduction de variables illustratives ( $c f$. note 7) offre des résultats intéressants (cf. Tableau B en annexe). Notamment la variable « densité » fait apparaître une corrélation significative et négative avec la composante 1, mais c'est là surtout le signe de l'opposition avec des grands pays, de faible densité, tels que les États-Unis, l'Australie, le Canada. De même, la variable «part de l'emploi agricole » est très bien corrélée avec la composante 3 (négativement), comme le sont la Grèce, le Portugal, les deux pays qui présentent les parts d'emploi agricole les plus élevées. Pour ne pas surcharger les graphiques, ces variables illustratives n'ont pas été représentées.
} 


\subsection{Les résultats de la classification en nuées dynamiques}

La CND a repris les mêmes variables et le même sous-ensemble de pays de l'OCDE. Plusieurs typologies ont été testées (en 5, 6, 7, 8 et 9 classes). Celle en 6 classes nous semble offrir les résultats les plus intelligibles; elle a donc été privilégiée. On la retrouve dans le tableau 2.

Tableau 2 : Classification des 21 pays en 6 classes

\begin{tabular}{|l|l|}
\hline 1 & USA \\
\hline 2 & Belgique, Japon, Norvège, Pays-Bas \\
\hline 3 & Allemagne, Autriche, Corée, Finlande, France \\
\hline 4 & Australie, Canada, Espagne, Grèce, Italie, Portugal \\
\hline 5 & Irlande, Royaume Uni \\
\hline 6 & Danemark, Suisse, Suède \\
\hline
\end{tabular}

Ce tableau révèle à la fois des regroupements plutôt attendus et d'autres davantage surprenants. La position atypique des USA est, de nouveau, visible. Ils forment à eux seuls une classe particulière. La connaissance des centres de classes en fonction des différentes variables (voir tableau $\mathrm{C}$ en annexe) confirme sa double caractéristique de pays le plus polluant et à la fiscalité environnementale la moins significative. La classe 6 (Danemark, Suisse, Suède) est caractérisée, au contraire, par les pollutions les plus faibles et un poids relativement élevé de la fiscalité. Les pays méditerranéens se retrouvent dans la même classe que l'Australie et le Canada ${ }^{14}$ (classe 4) : des situations proches en termes de pollutions et de consommations d'eau, en l'occurrence plutôt élevées, et des niveaux de taxes relativement faibles semblent pouvoir l'expliquer. L'Irlande partage la classe 5 avec le Royaume Uni : une variable joue un rôle important, à cet égard, celle des déchets municipaux et de la mise en décharge qui prennent des valeurs élevées. L'Europe continentale est scindée en 2 classes : la Belgique et les Pays-Bas, d'un côté (avec le Japon et la Norvège), l'Allemagne, l'Autriche, la France, de l'autre (avec la Finlande et la Corée). Les premiers font état d'un profil environnemental plutôt plus vertueux que la moyenne (notamment oxydes d'azote, oxydes de soufre, monoxyde de carbone relativement modérés), les autres (classe 3) sont dans une situation moyenne.

\subsection{Rapprochement avec les typologies générales}

C'est avec le modèle des « cinq capitalismes » d'Amable (2005) que nous proposons de rapprocher notre typologie relative à l'environnement et aux politiques environnementales. Il y a, certes, une forme d'arbitraire dans une telle option, si ce n'est que le modèle général en question est l'un des plus aboutis et l'un des mieux argumentés à l'aide de traitements statistiques (analyse de données et économétrie). Nous avons brièvement décrit plus haut ces cinq familles de capitalisme et précisé que le classement ne s'appliquait pas parfaitement selon les domaines concernés (marché de produits, marché du travail, finance, protection sociale, éducation). Nous n'y revenons pas.

La comparaison avec notre propre domaine de l'environnement fait apparaître des convergences mais aussi des différences non négligeables.

\footnotetext{
${ }^{14}$ Il faut aller jusqu'à une typologie de 9 classes pour que Canada et Australie se distinguent des autres.
} 
Dans la typologie d'Amable, « un groupe de pays ressort nettement comme spécifique et homogène : les économies anglo-saxonnes, représentant le modèle de capitalisme libéral de marché » (Amable, 2005, p. 226). Il s'agit des USA, du Royaume Uni, du Canada et de l'Australie. Dans notre classement, les USA constituent un cas très spécifique, eu égard notamment à l'importance des pollutions et à la faiblesse des taxes environnementales. Le Canada et l'Australie sont dans une autre catégorie qui comprend aussi les pays méditerranéens. Cependant, l'ACP qui précédait a permis de montrer aussi une certaine proximité entre ces deux pays et les USA sur l'axe 1. Le même positionnement au regard du libéralisme expliquerait-il cette situation particulière ou faut-il y voir le rôle d'autres caractéristiques, par exemple des densités de population plutôt faibles qui favoriseraient des déplacements nombreux et sur de longues distances (voir note $n^{\circ} 13$ ) ? Nous manquons d'éléments pour trancher.

Le Royaume Uni se retrouve, dans notre typologie, dans la même classe que l'Irlande, une situation qu'on retrouve chez Amable pour le marché des produits et la protection de l'emploi.

Le groupe des pays méditerranéens est assez homogène selon les axes factoriels et la CND, si ce n'est cette proximité assez surprenante avec le Canada et l'Australie que l'on vient d'indiquer. Le tableau de données brutes permet de voir en effet des profils assez proches pour certaines pollutions (niveaux d'oxydes de soufre) et la consommation d'eau (élevée en raison du poids de l'agriculture), mais aussi des différences (des taux bien plus élevés de $\mathrm{CO}_{2}$ pour l'Australie et le Canada) qui feraient rompre ce groupe si la typologie retenait un nombre plus élevé de classes.

Le capitalisme social-démocrate qu'Amable identifie à la Suède, au Danemark et à la Finlande est observable partiellement dans notre analyse : en effet, une classe contient bien les deux premiers pays mentionnés, mais la Finlande n'en fait pas partie, tandis que la Suisse en est une composante. La Suisse fait, en effet, apparaître un profil de pollution voisin des deux nations scandinaves, tandis que la Finlande est globalement plus polluante.

Ce dernier pays figure alors dans une classe où sont présents des pays représentatifs du capitalisme européen continental (Allemagne, Autriche, France), mais avec en plus la Corée. Chez Amable, le voisinage de l'Allemagne, de l'Autriche et de la France s'obtient pour le domaine de la protection sociale (avec la Belgique et la Suisse) (Amable, 2005, p. 203) : difficile de faire, pour autant, une analogie avec les politiques environnementales, car des différences significatives sont à remarquer entre ces trois pays, pour ce qui concerne la taxation ou la politique de recyclage.

La dernière classe $\left(\mathrm{n}^{\circ} 2\right.$ dans notre typologie) reprend le reste des pays du capitalisme européen continental, auxquels s'ajoute le Japon. On a déjà dit que ces pays étaient plutôt caractéristiques d'un profil environnemental plus satisfaisant que la moyenne.

Ainsi, même si des agencements très particuliers marquent les résultats de notre travail de classification des 21 pays de l'OCDE, pour autant la configuration d'Amable est loin d'être invalidée, dans l'hypothèse d'une application au domaine l'environnement et des politiques environnementales ${ }^{15}$. Des parties homogènes de cette configuration sont tout à fait visibles : pays méditerranéens, pays du capitalisme européen continental en deux blocs, proximité social-démocrate Danemark-Suède, proximité libérale Canada-

\footnotetext{
${ }^{15}$ Pour le moins, il ne nous semble pas exister un quelconque modèle de « capitalisme environnemental » qui s'opposerait ou s'ajouterait aux cinq capitalismes d'Amable.
} 
Australie, caractère singulier des USA. Il est probable que d'autres variables jouent sur les positionnements - nous pensons à la densité de population -, mais l'hypothèse d'une influence politique et culturelle semble validée par nos résultats. De manière plus précise, nous serions tentés de lire dans le positionnement des pays selon l'environnement, le jeu de la complémentarité institutionnelle évoquée plus haut et qui s'appliquerait ainsi au domaine de l'environnement et des politiques environnementales. La caractéristique de système libéral jouant dans le sens d'un interventionnisme limité trouverait à s'illustrer dans une fiscalité écologique faible ; le souci de bien collectif fortement présent dans le modèle social-démocrate se traduirait par des niveaux de pollutions moindres qu'en moyenne, etc. Il serait évidemment prématuré de conclure définitivement sur ce point, mais il y a là une piste à considérer avec attention.

\section{Perspectives et conclusion}

Ce premier travail, qui a bénéficié de l'apport des méthodes d'analyse de données, est à poursuivre pour mieux établir les rapprochements/différences entre pays. Vu l'ampleur de la tâche, c'est un programme de recherche à part entière qui semble nécessaire. Il aurait notamment pour tâche d'expliciter les caractéristiques des politiques environnementales et leur histoire, et plus largement, des rapports économiques à l'environnement. Une succession d'investigations monographiques s'avérerait alors des plus utiles. La recherche de liens entre la structuration des rapports à l'environnement et les traits des modes de régulation et formes institutionnelles serait un autre moment important de cette démarche de recherche. Dans une perspective de recherche de complémentarité institutionnelle, il s'agirait alors de voir si des facteurs communs (par exemple des valeurs culturelles typiques) ou des caractéristiques de «path dependency» institutionnelle sont de nature à influer à la fois sur les situations/politiques environnementales et sur l'une ou l'autre des formes institutionnelles.

Dans l'immédiat, le travail d'analyse de données n'a pas non plus réalisé toutes ses potentialités. Des investigations complémentaires pourraient être menées qui suivraient deux orientations particulières :

un élargissement du nombre de pays considérés, allant notamment jusqu'à l'ensemble des 30 pays de l'OCDE. Dans le présent texte, nous avons délibérément limité le nombre de pays pour permettre une comparaison avec la typologie générale d'Amable, mais il serait également intéressant de réaliser le travail de classification sans recourir à une référence particulière et de voir si, par exemple, les pays d'Europe de l'est font apparaître un profil similaire ou si la Turquie et le Mexique se rapprochent de l'ensemble ou s'en distinguent nettement.

une augmentation du nombre de variables, qu'elles aient trait aux situations environnementales proprement dites ou aux politiques environnementales. En l'occurrence, deux prolongements d'ordre méthodologique sont à mentionner. D'une part, la possible intégration de caractéristiques qualitatives (notamment utilisation de tel ou tel outil de politique environnementale sans référence au poids quantifié de cette utilisation) obligerait à se tourner vers des méthodes différentes, en particulier l'analyse des correspondances multiples. D'autre part, il serait intéressant de bien distinguer les analyses portant sur les variables environnementales et celles ayant trait spécia- 
lement aux politiques environnementales. Cette façon de traiter à part les formes institutionnelles de l'environnement serait, en vérité, plus propice à l'examen de l'hypothèse de complémentarité institutionnelle.

\section{Références}

Amable B., 2005. Les cinq capitalismes - Diversité des systèmes économiques et sociaux dans la mondialisation. Le Seuil, Paris.

Amable B., Barré B., Boyer R., 1997. Les Systèmes d'innovation à l'ère de la globalisation. Economica, Paris.

Aoki M., 1994. The contingent governance of teams: analysis of institutional complementarity. International Economic Review 35, 657-676.

Aoki M., 2001. Towards a comparative institutional analysis. MIT Press, Cambridge (Mass.).

Becker J., Raza W. G., 2000. Theory of regulation and political ecology : an inevitable separation ? Économies et Sociétés, Série « Théorie de la régulation »11, 55-70.

Benhaim J., Caron A., Levarlet F., 1991. Analyse économique des propositions des acteurs face au CO2. Cahiers du $C 3 E$, Université de Paris 1.

Benton T., 1989. Marx and natural limits: an ecological critique and reconstruction. New Left Review 178, 51-86.

Boyer R., 2002. Variété du capitalisme et théorie de la régulation. L'Année de la Régulation 6, 125-194.

Boyer R., Saillard Y. (eds), 1995. Théorie de la Régulation : l'état des savoirs. La Découverte, collection « Recherches », Paris.

Gendron C., 2001. Éthique et Développement Économique : le Discours des Dirigeants sur l'Environnement. Thèse de doctorat, Université du Québec, Montréal, édité en 2006, Le Développement durable comme compromis - La modernisation écologique de l'économie à l'ère de la mondialisation. Presses de l'Université du Québec, Québec.

Görg C, Brand U, 2000. Global environmental politics and competition between nation-states: on the regulation of biological diversity. Review of International Political Economy 7 (3), 371-398.

Gibbs D., 1996. Integrating sustainable development and economic restructuring: a role for regulation theory. Geoforum 27, 1-10.

Gibbs D., 2006. Prospects for an environmental economic geography: linking ecological modernisation and regulationist approaches. Economic Geography 82, 193-215.

Lipietz A., 1995. Écologie politique régulationniste ou économie de l'environnement ? R. Boyer, Y. Saillard (eds), Théorie de la Régulation : l'état des savoirs. La Découverte, collection « Recherches », Paris.

Lipietz A., 1997. The post-Fordist world: labour relations, international hierarchy and global ecology. Review of International Political Economy 4, 1-41.

McManus P, 2002. The potential and limits of progressive neopluralism: a comparative study of forest politics in Coastal British Columbia and South East New South Wales during the 1990s. Environment and Planning A 34 (5), 845- 865.

OCDE, 2007. Données OCDE sur l'environnement, Compendium 2006/2007.

Rousseau S., 2002. Économie et environnement : une analyse régulationniste de la rente environnementale. Thèse de doctorat en sciences économiques, Université de Lille 1.

Rousseau S., Zuindeau B., 2007. Théorie de la régulation et développement durable. La Revue de la Régulation 1, Varia, mis en ligne le 15 mai, http ://regulation.revues.org/document1298.html

Théret B., 1997. Méthodologie des comparaisons internationales, approches de l'effet sociétal et de la régulation : fondements pour une lecture structuraliste des systèmes nationaux de protection sociale. L'Année de la régulation $1,163-228$.

Théret, B., 2000. Nouvelle économie institutionnelle, économie des conventions et théorie de la régulation : vers une synthèse institutionnaliste ? La lettre de la régulation 35, 1-4. 
Zuindeau B., 2001. L'analyse des externalités environnementales : éléments pour un programme de recherche régulationniste. Géographie, Économie, Société 3, 71-92.

Zuindeau B., 2007. Regulation School and environment: theoretical proposals and avenues of research. Ecological Economics 62 (2), 281-290.

Zuindeau B., 2009. Analyse régulationniste de l'environnement : éléments théoriques et pistes de recherche. Économie appliquée 61(3), 159-188.

Nous tenons à remercier les deux rapporteurs anonymes dont les commentaires ont aidé à préciser un certain nombre de développements du texte. Les limites et les erreurs éventuelles demeurent évidemment de notre seule responsabilité. 


\section{ANNEXE}

Tableau A : tableau des données

\begin{tabular}{|c|c|c|c|c|c|c|c|c|c|c|c|}
\hline & $\mathrm{CO}_{2}$ & $\begin{array}{l}\text { Oxydes } \\
\text { de soufre }\end{array}$ & $\begin{array}{l}\text { Oxydes } \\
\text { d'azote }\end{array}$ & $\mathrm{CO}$ & Eau & $\begin{array}{l}\text { Déchets } \\
\text { municipaux }\end{array}$ & Décharge & Recyclage & $\begin{array}{c}\text { Déchets } \\
\text { nucléaires }\end{array}$ & Taxe 1 & Taxe 2 \\
\hline Canada & 17,49 & 76,3 & 78,4 & 290,2 & & 409,99 & 109,80 & & 4,0 & 1,2 & 3,7 \\
\hline USA & 19,68 & 49,4 & 63,9 & 272,7 & 1730 & 744,37 & 176,96 & 403,94 & 1,1 & ,9 & 3,5 \\
\hline Japon & 9,41 & 6,7 & 15,8 & 23,3 & 680 & 425,51 & 71,67 & 14,58 & 1,6 & 1,7 & 6,4 \\
\hline Corée & 9,36 & 10,4 & 24,4 & 17,0 & 550 & 377,91 & 185,83 & 137,50 & 3,0 & 2,8 & 11,4 \\
\hline Australie & 17,35 & 126,9 & 84,2 & 199,8 & 930 & 432,08 & 131,08 & 300,99 & 0 & 1,7 & 5,5 \\
\hline Autriche & 9,22 & 4,4 & 24,7 & 87,5 & 470 & 553,97 & 147,07 & 37,43 & 0 & 2,7 & 6,3 \\
\hline Belgique & 11,57 & 14,6 & 26,3 & 65,3 & 650 & 436,86 & 135,86 & 50,53 & 1,9 & 2,3 & 5,1 \\
\hline Danemark & 10,43 & 4,5 & 35,5 & 112,8 & 120 & 665,81 & 170,22 & 33,86 & 0 & 4,8 & 9,8 \\
\hline Finlande & 13,93 & 16,4 & 40,5 & 97,1 & 450 & 450,73 & 135,75 & 270,17 & 1,9 & 3,3 & 7,4 \\
\hline France & 6,33 & 9,0 & 22,7 & 92,8 & 560 & 553,57 & 87,69 & 199,47 & 4,1 & 2,1 & 4,9 \\
\hline Allemagne & 10,35 & 7,4 & 17,2 & 48,9 & 430 & 588,02 & 194,88 & 104,14 & 1,4 & 2,5 & 7,3 \\
\hline Grèce & 8,57 & 46,3 & 28,9 & 96,7 & & 422,46 & 34,26 & 388,20 & 0 & 2,3 & 6,5 \\
\hline Irlande & 10,28 & 24,5 & 31,0 & 53,8 & & 672,41 & 227,68 & 444,73 & 0 & 2,5 & 8,2 \\
\hline Italie & 7,80 & 11,5 & 21,8 & 65,1 & 730 & 542,09 & & 294,77 & 0 & 3,0 & 7,2 \\
\hline Pays-Bas & 11,38 & 5,3 & 26,6 & 36,7 & 560 & 621,62 & 157,90 & 10,71 & 0,1 & 3,6 & 9,5 \\
\hline Norvège & 7,84 & 4,9 & 46,9 & 96,5 & 750 & 373,88 & 125,91 & 97,00 & 0 & 3,0 & 6,8 \\
\hline Portugal & 5,64 & 28,4 & 27,8 & 58,5 & 860 & 473,22 & 40,62 & 303,26 & 0 & 3,0 & 8,7 \\
\hline Espagne & 7,68 & 37,3 & 34,7 & 53,8 & 900 & 515,91 & 46,20 & 266,68 & 1,5 & 2,0 & 5,6 \\
\hline Suède & 5,98 & 6,5 & 27,1 & 66,7 & 300 & 478,69 & 162,32 & 23,13 & 3,8 & 2,9 & 5,7 \\
\hline Suisse & 5,96 & 2,3 & 11,4 & 47,4 & 350 & 648,72 & 219,80 & 3,21 & 2,1 & 2,2 & 7,7 \\
\hline Royaume-Uni & 9,10 & 16,9 & 26,8 & 40,1 & 250 & 578,95 & 100,68 & 372,34 & 4,0 & 2,6 & 7,3 \\
\hline
\end{tabular}

(Source : COMPENDIUM sur l'environnement de l'OCDE).

Pour les ACP, les valeurs manquantes ont été remplacées par les valeurs moyennes des 21 pays considérés. 
Tableau B : Coordonnées des 11 variables sur les trois premières composantes

\begin{tabular}{|c|c|c|c|}
\hline \multirow[t]{2}{*}{ VARIABLES } & \multicolumn{3}{|c|}{ Coordonnées sur les trois premières composantes } \\
\hline & Composante 1 & Composante 2 & Composante 3 \\
\hline \multicolumn{4}{|l|}{ Variables actives } \\
\hline Emissions de $\mathrm{CO}_{2}$ par habitant\& & 0,795 & 0,367 & 0,157 \\
\hline Emissions d'oxydes de soufre par habitant & 0,856 & $-0,040$ & $-0,197$ \\
\hline Emissions d'oxydes d'azote par habitant & 0,876 & 0,122 & 0,024 \\
\hline Emissions de monoxyde de carbone par habitant & 0,883 & 0,169 & 0,183 \\
\hline Utilisation des ressources en eau douce par habitant & 0,725 & 0,124 & $-0,259$ \\
\hline Déchets municipaux par habitant & $-0,071$ & 0,746 & 0,194 \\
\hline Déchets mis en décharge par habitant & 0,515 & $-0,070$ & $-0,461$ \\
\hline Recyclage des déchets par habitant & $-0,185$ & 0,748 & 0,487 \\
\hline Déchets nucléaires & 0,022 & $-0,492$ & 0,736 \\
\hline Taxes relatives à l'environnement par rapport au PIB & $-0,730$ & 0,294 & $-0,259$ \\
\hline $\begin{array}{l}\text { Taxes liées à l'environnement par rapport aux } \\
\text { recettes fiscales }\end{array}$ & $-0,716$ & 0,313 & $-0,319$ \\
\hline \multicolumn{4}{|l|}{ Variables illustratives* } \\
\hline PIB par habitant & 0,267 & 0,449 & 0,438 \\
\hline Part de l'emploi agricole & $-0,124$ & $-0,232$ & $-0,725$ \\
\hline Densité démographique & $-0,517$ & $-0,005$ & 0,071 \\
\hline
\end{tabular}

* elles n'entrent pas dans la construction des composantes

Tableau C : Classification en nuées dynamiques (en 6 classes)

\begin{tabular}{|c|c|c|c|c|c|c|}
\hline \multicolumn{7}{|c|}{ Centres finaux des classes } \\
\hline & \multicolumn{6}{|c|}{ Classe } \\
\hline & 1 & 2 & 3 & 4 & 5 & 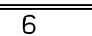 \\
\hline $\mathrm{CO} 2$ & 19,68 & 10,05 & 9,84 & 10,76 & 9,69 & 7,46 \\
\hline oxydes de soufre & 49,4 & 7,9 & 9,5 & 54,5 & 20,7 & 4,4 \\
\hline oxydes d'azote & 63,9 & 28,9 & 25,9 & 46,0 & 28,9 & 24,7 \\
\hline $\mathrm{CO}$ & 272,7 & 55,5 & 68,7 & 127,4 & 47,0 & 75,6 \\
\hline eau & 1730 & 660 & 492 & 855 & 250 & 257 \\
\hline Déchets municipaux & 744,37 & 464,47 & 504,84 & 465,96 & 625,68 & 597,74 \\
\hline Recyclage & 176,96 & 122,83 & 150,24 & 72,39 & 164,18 & 184,11 \\
\hline mise en décharge & 403,94 & 43,20 & 149,74 & 310,78 & 408,54 & 20,06 \\
\hline Déchets nucléaires & 1,1 & ,9 & 2,1 & 9 & 2,0 & 2,0 \\
\hline Taxes 1 & 9 & 2,7 & 2,7 & 2,2 & 2,6 & 3,3 \\
\hline Taxes 2 & 3,5 & 7,0 & 7,5 & 6,2 & 7,8 & 7,7 \\
\hline route & 15,9 & 7,5 & 7,2 & 7,7 & 8,5 & 8,4 \\
\hline
\end{tabular}

